\title{
A pillar-based microfluidic chip for T-cells and B-cells isolation and detection with machine learning algorithm
}

\author{
Bilal Turan' ${ }^{1}$,Taisuke Masuda ${ }^{*}$, Wu Lei ${ }^{2}$, Anas Mohd Noor ${ }^{3}$, Koji Horio ${ }^{1}$, Toshiki I. Saito ${ }^{4}$, Yasuyuki Miyata ${ }^{4}$ \\ and Fumihito Arai ${ }^{1}$
}

\begin{abstract}
Absolute counting of total leukocytes and specific subset (such as T-cells and B-cells) within small amounts of whole blood is difficult due to the lack of techniques that enables separation of leukocytes from limited volume of whole blood. In this study, a microfluidic chip equipped with a size controlled micropillar array for highly separation of T-cells and B-cells from sub-microliter of whole blood was studied. Based on the difference in size and deformability, leukocytes were separated from other blood cells by micropillar arrays. However, the variability of cells in size, morphology and color intensity along with the spectrum crosstalk between fluorescence dyes make cell detection among pillars extremely difficult. In this paper, an support vector machine supervised machine learning classifier based on both Histogram of Oriented Gradients (HOG) and color distribution features was proposed to distinguish T-cells and B-cells fast and robustly. HOG features were utilized to detect cells from background and noise; color distribution features were employed to alleviate the effect of fluorescence spectrum crosstalk. Experiment showed we achieved average detection accuracy of 94\% for detecting T-cells and B-cells from the background. Furthermore, we also got 96\% accuracy with cross validation to detect T-cells from B-cells. Both theoretical analysis and experiments demonstrated the proposed method and system has high performance in T-cells and B-cells counting. And our microfluidic cell counting system has great potential as a tool for point-of-care leukocyte analysis system.
\end{abstract}

Keywords: Histogram of Oriented Gradients features, Microfluidic chip, Machine learning, Support vector machine, T-cells, B-cells

\section{Introduction}

Leukocytes and their subtypes population numbers change dramatically in the presence of infections, malignancies, autoimmune disorders, and chemical-induced hematotoxicity. The two categories of lymphocytes, $\mathrm{T}$-cells and B-cells, are important constituents of the human immune system as their concentration, quantified by a count test, indicates the state of body's immune response against infections [1]. Currently, fluorescenceactivated cells sorting (FACS) is used as golden standard

\footnotetext{
*Correspondence: masuda@mech.nagoya-u.ac.jp

${ }^{1}$ Department of Micro-Nano Mechanical Science and Engineering, Graduate School of Engineering, Nagoya University, 1 Furo-cho, Chikusa-ku, Nagoya 464-8603, Japan

Full list of author information is available at the end of the article
}

in biological research and clinical diagnostics to automatically determine the count or concentration for one or multiple types of cells. However, requirements of large volume sample (in the order of $\mathrm{mL}$ ) and expensive reagent, bulky equipment size, sophisticated costly optical component and the requirement of technical personnel make conventional FACS prohibitive for point-of-care application. In addition, FACS is traditionally relied on non-imaging technique by laser scattering and fluorescence emission for cell identification and hence there is no image information of cells [2]. There is a tremendous need for an automated, portable point-of-care blood cell counter that could yield results in a matter of minutes from a drop of blood without any trained professionals to operate the instrument [3]. This technology could 
potentially have tremendous applications in hospitals, private clinics, retail clinics and the developing world.

In order to develop a compact leukocyte counting system, researchers are actively looking toward microfluidic devices as the platform for the next generation translatable cell sorter. Microfluidic based devices are a proven technology for cellular handling as they can offer precise spatial and temporal control in a greatly miniaturized platform $[4,5]$. For cell isolation, there are several methods that utilize the physical characteristics of the cell [6]. Blood cells can be separated using non-inertial forces and concepts such as magnetic bead immunoassay [7], dielectric properties [8], optical force [9], magnetic [10], electrohydrodynamic [11], or acoustic $[12,13]$, as active separation strategies. The non-inertial forces may have some unexpected physiological effects on the blood component properties which are unsuitable for the downstream application of the separated products. These techniques add to the complexity of the microfluidic devices and normally run at very low flow rates. On the other hand, passive separation techniques exploit the difference in size and deformability of cells in which they do not involve external forces but rely purely on the interaction of the blood with internal geometries of microfluidic devices [14]. The pore-based or pillar-based passive devices have simple mechanical design and can function in wide range of flow rates $[15,16]$, WBCs are flexible and can deform to pass through $7 \mu \mathrm{m}$ pores. The RBCs are more flexible and can fold or align to cross the filter opening of 3-4 $\mu \mathrm{m}$ [17]. A perfect blood microfilter can trap all WBCs and pass all RBCs continuously without loss of separation efficiency at different flow rates and cell concentration.

After isolation, captured cells are usually distinguished from microfluidic chip's fluorescent microscopy images. Using different cell markers, we can detect, identify and count cells. However, cells imaged by fluorescence microscopy exhibit heterogeneous intensity levels and are often badly contrasted. The variability of cell size and morphology, differences in illumination over time and across whole microfluidic chip hamper the ability to specify a global set of parameters for cell detection algorithms over the whole experiment. Furthermore, there are serious spectrum crosstalk between fluorescence dyes when performing dual or multiple cells detection. The available algorithms for illumination correction and segmentation do not perform well enough to achieve satisfying results on many experiments [18]. Therefore, there is a strong motivation for the development of an automatic cell detection and counting method [19]. A machine learning algorithm, with an appropriate feature extractor for the application is the key to success. For this, Histogram of Oriented Gradients (HOG) represents a robust feature descriptor used in computer vision area for object detection [20]. One of the key advantages of HOG is being able to describe object orientation while showing invariance to geometric and photometric transformations because it operates in localized regions. In other words, HOG tends to be unaffected by changes in shapes and lighting, which appear in larger spatial regions. These advantages make HOG features fit our situation especially well due to the cells variability in intensity and shape on pillar based microfluidic chip. A support vector machine (SVM) can be used to classify HOG features. SVM is a supervised machine learning algorithm, which creates a model according to the training data, and then the test data can be classified according to said model [21].

In this study, we propose a pillar-based microfluidic chip and system with machine-learning for T-cells and B-cells isolation and detection. The design of micropillar array with gradually narrowed gaps were optimized to trap leukocytes while allowing other blood cells to flow through [22]. The size and deformability-based separation of leukocytes require micropillar of proper gap size and precise control of pressure applied to the array. Under optimized conditions, a sub-microliter of whole blood was suspended in a buffer and then directly introduced to the micropillar array without pre-treatment such as density gradient or RBCs lysis. All leukocyte types were individually trapped between micropillars, while other elements of blood passed through the micropillar array into a waste reservoir. To detect T-cells and B-cells from fluorescence microscopy images, a machine learning method based on both HOG and color features was studied.

\section{Materials and methods}

\section{Peripheral blood sample preparation}

Human blood samples were collected from healthy donors at National Hospital Organization Nagoya Medical Center. The study protocol was reviewed and approved by the institutional review board, and written informed consent was obtained from all participants. The samples were collected in a collection tube with EDTA to prevent coagulation and were used within $24 \mathrm{~h}$. For $\mathrm{T}$-cells and B-cells detection, blood sample was mixed with a two-color direct immunofluorescence reagent (BD Simultest TM CD3-FITC/CD19-PE, BD Bioscience, San Jose, $\mathrm{CA}$ ) and incubated in room temperature for $15 \mathrm{~min}$.

\section{A pillar-based microfluidic chip}

We designed a pillar-based microfluidic chip to isolate leukocytes from peripheral blood with high efficiency and without clogging [22]. Figure 1a shows the schematic layout of the proposed microfluidic filter. Blood is pumped into the device via inlet port, enters the filtration 


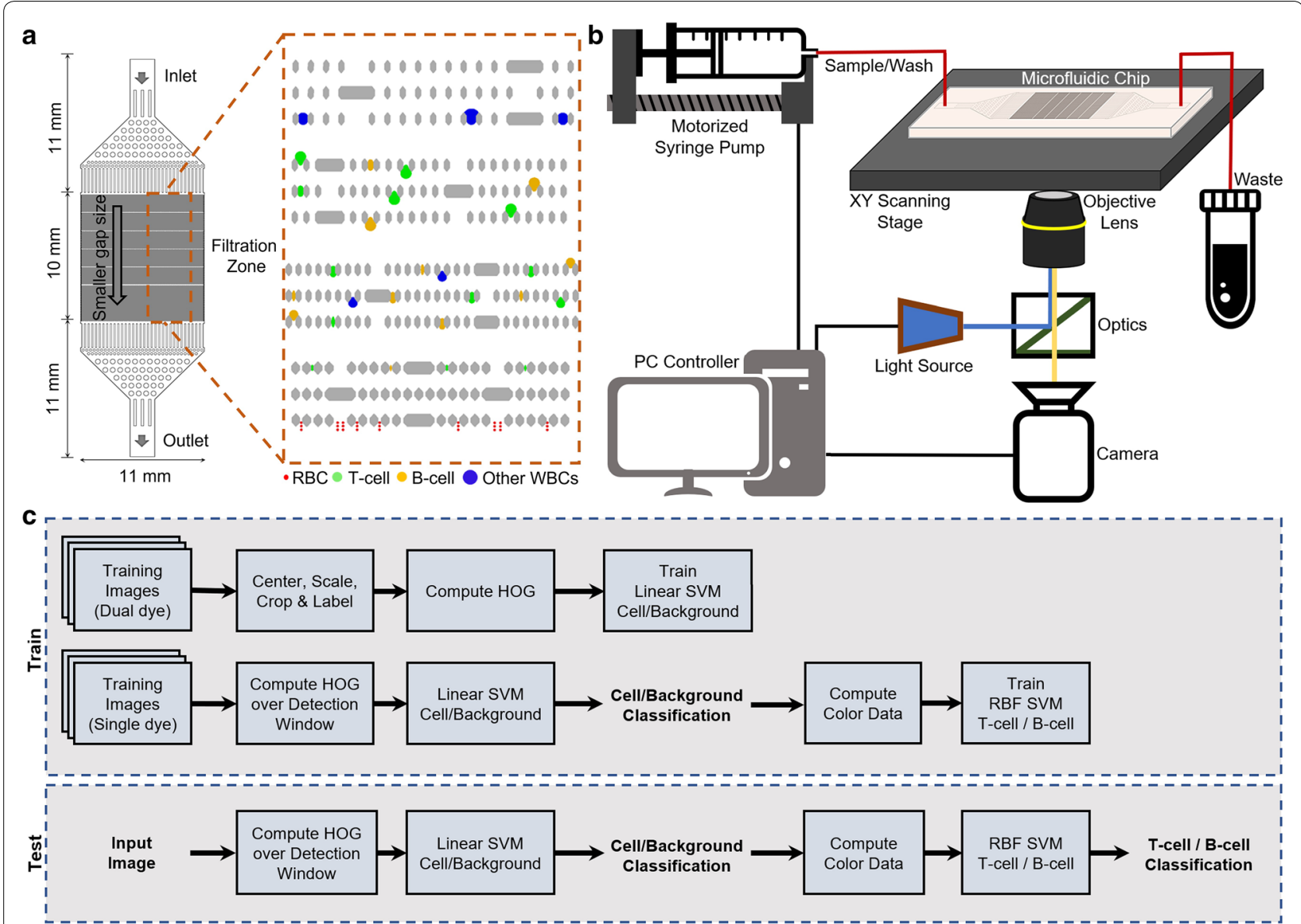

Fig. 1 Overview of the proposed system. a Design of the microfluidic chip, blood is pumped into the device via inlet port. Due to the difference in size and deformability, leukocytes will be trapped in different zones. $\mathbf{b}$ Experiment setup. $\mathbf{c}$ Block diagram of cell detection framework using machine learning method

zone and leaves it at outlet port. Outlet port is for collecting the components of blood passing the filter. Proposed microfluidic chip has 12 different groups of pillar arrays [22]. Main difference between these pillar arrays is the spacing between each pillar, which we call gap size. The gap size for these 12 pillar arrays are gradually decreasing in size from the inlet port to the outlet port, $15-3 \mu \mathrm{m}$ respectively. The smallest gap size, $3 \mu \mathrm{m}$, can prevent leukocytes flowing away while allow red blood cells or platelets to pass through. Larger and less deformable cells are captured by pillar arrays with bigger gaps while smaller cells flowed through, which makes this design suitable to prevent clogging. To further avoid clogging, escape routes without trap structure among pillars are designed, as Fig. 1a shows. The schematic and prototype of microfluidic chip was shown in Fig. 1b. The single inlet single outlet fabricated device was connected to a gastight syringe pump (1710 TLLX SYR, Hamilton, USA), which was manipulated by a high precision controller (QT-AMH2, Chuo Precision Industrial, Japan). The PTFE tubing was inserted into the Tygon tubes of the device fluidic interconnects and was used for pumping of sample to the inlet port of the device and transfer of separated components from the device outlet port to a collection tube. The whole experiments were monitored with an inverted optical microscope for detailed investigation of the filtration process. The images and videos were captured by a camera (ASI178 MC, Zhen Wang Optical Company, China, $3096 \times 2080$, pixel size $2.4 \mu \mathrm{m}$ ) installed on the eyepiece body tube of the microscope. The procedure of acquisition is semi-auto and the operator controls some camera parameters, such as, magnification, exposure time and focus. After sample introduction, the filtration zone on chip was scanned automatically frame by frame for counting trapped T-cells and B-cells. As described in sample preparation part, T-cells were stained by CD3-FITC (Ex $494 \mathrm{~nm}$, Em $520 \mathrm{~nm}$ ) excitation and B-cells were stained by CD19-PE (Ex 496 nm, Em 
$578 \mathrm{~nm}$ ). In the next section, we would present a method for detecting T-cells and B-cells in the images characterized in this section.

\section{Microfluidic chip fabrication}

Our microfluidic chip fabrication process is based on the soft-lithography technique. We used a Si-wafer to make a master mold. The microchannel part is made of polydimethylsiloxane (PDMS) by using a molding process. Then, the microchannel part is bonded to a glass plate to form the microfluidic chip. First, the photomask was prepared using laser lithography (DWL66FS, Heidelberg Instruments Mikrotechnik GmbH, Heidelberg, Germany). We then prepared the Si-wafer (thickness $500 \mu \mathrm{m}$, Matsuzaki Ltd., Japan) for spin coating with an epoxybased photoresist (SU-8 3010, Microchem, MA, USA) to the required thickness $(\sim 10 \mu \mathrm{m})$ and cured it using a soft bake $\left(95^{\circ} \mathrm{C}, 30 \mathrm{~min}\right)$ process. Next, the Si-wafer was exposed to UV light with the photomask using a mask aligner (Suss MA6, SUSS MicroTec, Germany). The Siwafer was developed and dry-etched by deep reactive ion etching (RIE-800, Samco, Japan). Plasma polymerization (RIE-800, Samco, Japan) of octafluorocyclobutane $\left(C_{4} F_{8}\right)$ was used for passivation of the $\mathrm{Si}$-mold to make the surface non-adhesive during repeated PDMS microfluidic chip demolding processes. The PDMS microchannel part was prepared using a PDMS pre-polymer (Silpot 184, Dow Corning Toray Co., Ltd., Japan) mixed in a $10: 1(\mathrm{w} / \mathrm{w})$ ratio with a curing agent, then pouring onto the Si-mold and baking at $85^{\circ} \mathrm{C}$ for $45 \mathrm{~min}$. The cured PDMS was then demolded and the PDMS microchannel part was treated with an $\mathrm{O}_{2}$ plasma (Femto Science CuteMPR, South Korea) to complete the PDMS-glass bonding process.

\section{Cell detection}

The framework of object detection with HOG and color features is illustrated by the block diagram in Fig. 1c. It consists of separate training and testing phases. In the training phase, we trained two separate SVMs to detect cells from background and to identify the detected cells as T-cells or B-cells. First using the dataset that is generated from the dual dyed images, a linear-kernel SVM is trained to detect cells from background. Then, the images that is dyed with only singe dye is exhaustively searched using sliding window method, finding HOG for each window and classified by the first SVM. Found cells then used for their color information to train a Radial Basis Function (RBF)-kernel SVM [21] to identify T-cells from B-cells. Although RBF-SVM is timewise costly, it gives better accuracy. In the testing phase, unseen images are scanned with sliding window and HOG features are generated from the position of each window in the image.
These features are classified as cell or background with the linear SVM classifier, then classified cells are further processed to get the color information and classified as T-cells or B-cells using the RBF SVM.

The HOG and color-based features extraction will be described in the next section.

\section{Results}

In order to get an idea about how well the T-cells and B-cells are detected, it is necessary to study both SVM classifier and performance of the system as a whole.

\section{Cell isolation}

Cell isolation experiment is performed for several times, to test the performance of our system and method. The experiment condition is decided empirically [22]. First, we introduced sheath liquid (PBS with $5 \mathrm{mM}$ EDTA) into the microfluidic chip to remove the bubbles. We introduced $1 \mu \mathrm{L}$ peripheral blood into chip and collected the fluid out of chip in a tube. After all blood sample flowed through the chip, $30 \mu \mathrm{L}$ sheath liquid was introduced to remove non-trapped cells. The flow rates for peripheral blood and sheath liquid are both set to be $10 \mu \mathrm{L} /$ min. Then, the whole filtration part on chip was scanned frame by frame with the control of motorized stage. When scanning, T-cells and B-cells are excited (488 nm). 420 frames were captured for the whole filtration zone. Figure 2 shows partial scanned images of the filtration zone. According to the design principle, larger cells were mostly trapped in wide pillar gaps, while smaller cells, such as T-cells and B-cells, were mainly trapped in narrow pillar gaps. As Fig. 2 shows, most T-cells (green) and B-cells (yellow) were captured in 5-8 $\mu$ m gaps area.

\section{Dataset and methodology}

There is no standard dataset for fluorescent cell images, so we created a custom dataset to be used in our detection algorithms. To train and test the detection of cells we used the scan images from 10 experiments that is dyed with the dual dye. Seven experiments are used as training data which consists of 2940 images. Another three experiments which consists of 1320 images are reserved for test. A total of 6200 cell images and 35,000 background images are gathered semi-automatically from the training images. Figure 3 shows a sample from this dataset.

Cells come in many sizes and shapes since they are deformed by the pillars of the microfluidic chip. To simplify the problem we scaled each cell, so the detector only needs to be trained for the shape information. First a small set of cell images are cropped manually from the training images. This is used to train the initial detector which is then used to automatically detect and gather training images time effectively. Found cells are 


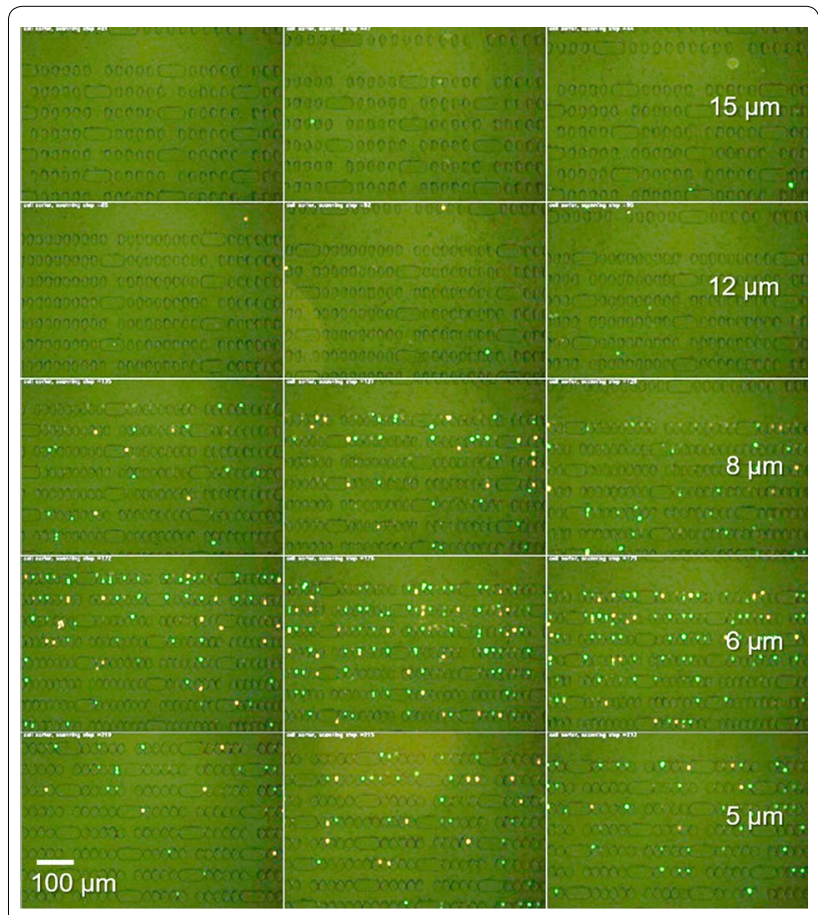

Fig. 2 Partial scanned images of the filtration zone for T and B lymphocytes

then cropped and scaled. Then all the cropped images are manually checked to eliminate the misdetections and noises, which are used as hard negatives as Fig. 3b shows. After this semi-automated process, 50 training images which are dense in cells are annotated manually, also fixing the scaling of the cells when necessary. Negative training samples are then automatically cropped in different scales from these annotated images. Using the positive and negative training data from this semi-automatic process the detector is trained, then annotated images are exhaustively searched for misdetections. Found hard negatives are added to the training dataset to re-train the final detector.

Crosstalk between the dyes, prevents us from manually annotating the datasets to be used as training data. Hence in order to train the second SVM which identifies T-cells from B-cells, we used the datasets which are only dyed with one color.

\section{HOG descriptor and color distribution features}

Preparing the data set appropriately is vital for achieving good performance. Cell images are centered and scaled. Since the size of the cells vary, we upscaled them to 80 pixels squares, with the scaling factor appropriate to the longest side of the cell bounding boxes. A margin of 16 pixels around the cell on all four sides are included, as this border provides a significant amount of context that helps detection. As Fig. 4 shows, HOG helps us define the shape context of the cells effectively, which in return makes it possible to detect all different shapes of cells deformed by the micropillars in our study.

We computed HOG descriptors for the prepared cell samples. A HOG feature vector is modeled for such an image. Figure 4 shows step by step computation of HOG descriptors. First, one the image gradient values are determined, representing directional changes in the intensity or color. The gradient vector is formed by combining the partial derivatives of image $\mathrm{I}$ in the $\mathrm{x}$ and $\mathrm{y}$ directions,

$$
\nabla I=\sqrt{\left(\frac{\partial I}{\partial x}\right)^{2}+\left(\frac{\partial I}{\partial y}\right)^{2}}
$$

The gradients in the two directions are computed by applying the $1 \mathrm{D}$ centered, point discrete derivative mask in the horizontal and vertical directions,

$$
\begin{aligned}
& \frac{\partial I}{\partial x}=I \times\left[\begin{array}{lll}
-1 & 0 & 1
\end{array}\right], \\
& \frac{\partial I}{\partial y}=I \times\left[\begin{array}{c}
-1 \\
0 \\
1
\end{array}\right] .
\end{aligned}
$$

The gradient orientations are computed using the vertical and horizontal gradients as,

$$
\theta=\tan ^{-1}\left(\frac{\partial I}{\partial x} \div \frac{\partial I}{\partial y}\right)
$$

The image $I$ is then divided into cells, and for each cell, a local 1D histogram of gradient directions (orientations) is calculated over the pixels from that cell. We used nine bins for the local histogram. The histogram channels are evenly spread over $0^{\circ}-180^{\circ}$ in absolute values, so each histogram bin corresponds to a 20-degree orientation interval. The obtained cell histograms are then combined into a descriptor vector of the image. First, these cells locally contrast normalized, due to the variability of illumination and shadowing in the image. That requires grouping the cells together into larger, spatially-connected blocks. Once the normalization is performed, all the histograms can be concatenated in a single feature vector, representing the HOG descriptor. Depending on the cell size and block overlap, the amount of detail one could get changes. We used $[8 \times 8]$ cell size to calculate blocks of $[16 \times 16]$ pixels with overlaps using nine histogram channels. These setting can be thought as default choice in object detection. The feature vector of 


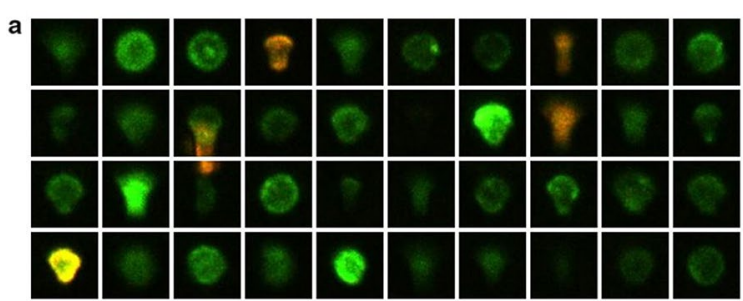

b
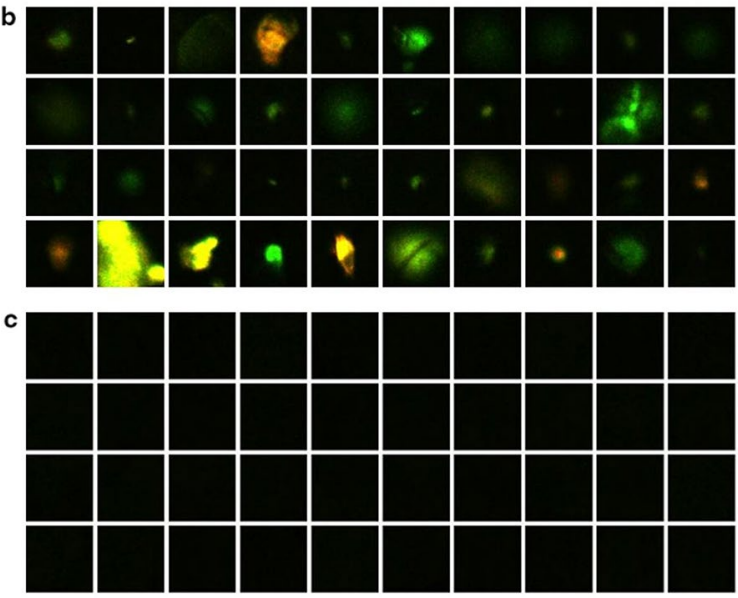

Fig. 3 Example images from the dataset. a Cell images centered and scaled. $\mathbf{b}$ Hard negative images, that is manually eliminated. c Background images

the image is computed as its HOG descriptor, with 2916 coefficients.

Because the long emission spectrum tail of dyes causes overlap like with the fluorophores FITC and PE, in our T-cells and B-cells counting experiment, cells are stained by a two color (CD3-FITC/CD19-PE) immunofluorescence dye. The color vectors for cells fluctuate in a big range. Cells can be sorted from background and noise through HOG features, but among the detected cells, it is difficult to decide the cell type when taking multicolor cell detection. To alleviate the influence of fluorescence emission spectrum crosstalk, we calibrated the color distribution of FITC and PE single dye stained cells and then classified dual stained cells by the model trained from single dye stained cells color features. We stained samples with single dye, FITC Mouse Anti-Human CD3 (BD Pharmingen $^{\mathrm{TM}}$, BD Bioscience, San Jose, CA) for T-cells and PE Mouse Anti-Human CD19 (BD Pharmingen ${ }^{\mathrm{TM}}$, BD Bioscience, San Jose, CA) for B-cells. The single dye stained samples were introduced to the same isolation system and sorted under the same condition as dual dye stained sample. As stained by only one fluorescent dye, the detected cells' type could be decided. We used previously trained HOG detector to detect the cells and extracted the average color in RGB space for every cell in FITC and PE. After the cells are detected, an adaptive thresholding using Otsu's method is performed to find the cell segment [23]. Then average color in RGB is calculated using this thresholding as a mask. We trained an RBF kernel SVM using the color averages from single dye stained cells. This calibration needs to be performed only once. Through cross-validation, an average accuracy of $96 \%$ was achieved.

\section{Detection results of $\mathrm{T}$-cells and $\mathrm{B}$-cells}

We have created a data set to test the performance of the HOG detector. 500 positive cell images are cropped, centered and scaled from the annotated test images. 50 difficult examples containing noise and 450 randomly selected background examples are used as negative test set. To quantify the HOG detector performance, we plot Receiver Operating Characteristics (ROC's), i.e. true positive rate $\left(\frac{\text { TruePos }}{\text { TruePos }+ \text { FalseNeg }}\right)$ versus false positive rate $\left(\frac{\text { FalsePos }}{\text { FalsePos }+ \text { TrueNeg }}\right)$. Using also this test set we calculated accuracy, specificity and sensitivity values as:

$$
\begin{aligned}
& \text { Accuracy }=\left(\frac{\text { TruePos }+ \text { TrueNeg }}{\text { Total Population }}\right) \\
& \text { Specificity }=\left(\frac{\text { TrueNeg }}{\text { TrueNeg }+ \text { FalsePos }}\right) \\
& \text { Sensitivity }=\left(\frac{\text { TruePos }}{\text { TruePos }+ \text { FalseNeg }}\right)
\end{aligned}
$$

In addition to this set of 1000 image patches, negative images from test data exhaustively searched and around 4,500,000 negative images are added to plot Detection Error Tradeoff (DET) curve on log-log scale i.e. miss rate $\left(\frac{\text { FalseNeg }}{\text { TruePos }+ \text { FalseNeg }}\right)$ versus false positive per window $\left(\frac{\text { FalsePos }}{\text { TotalPopulation }}\right)$. DET curves present the same informa-

tion as ROC's but small differences in probabilities are easier to distinguish. Figure 5 presents ROC's and DET curves.

Different points in ROC and DET curves corresponds to different accuracy, specificity and sensitivity. We have achieved an accuracy of $94 \%$ and sensitivity of $90 \%$ when specificity was chosen to be $99 \%$.

In one experiment, 420 frames are scanned for the filtration zone on the microfluidic chip. With the classifiers trained with HOG and color distribution feature, all the 420 images were processed in $67 \mathrm{~s}$ with our experiment PC (CPU i7-8700 K 3.7 GHz, Nvidia GTX 1080Ti, 

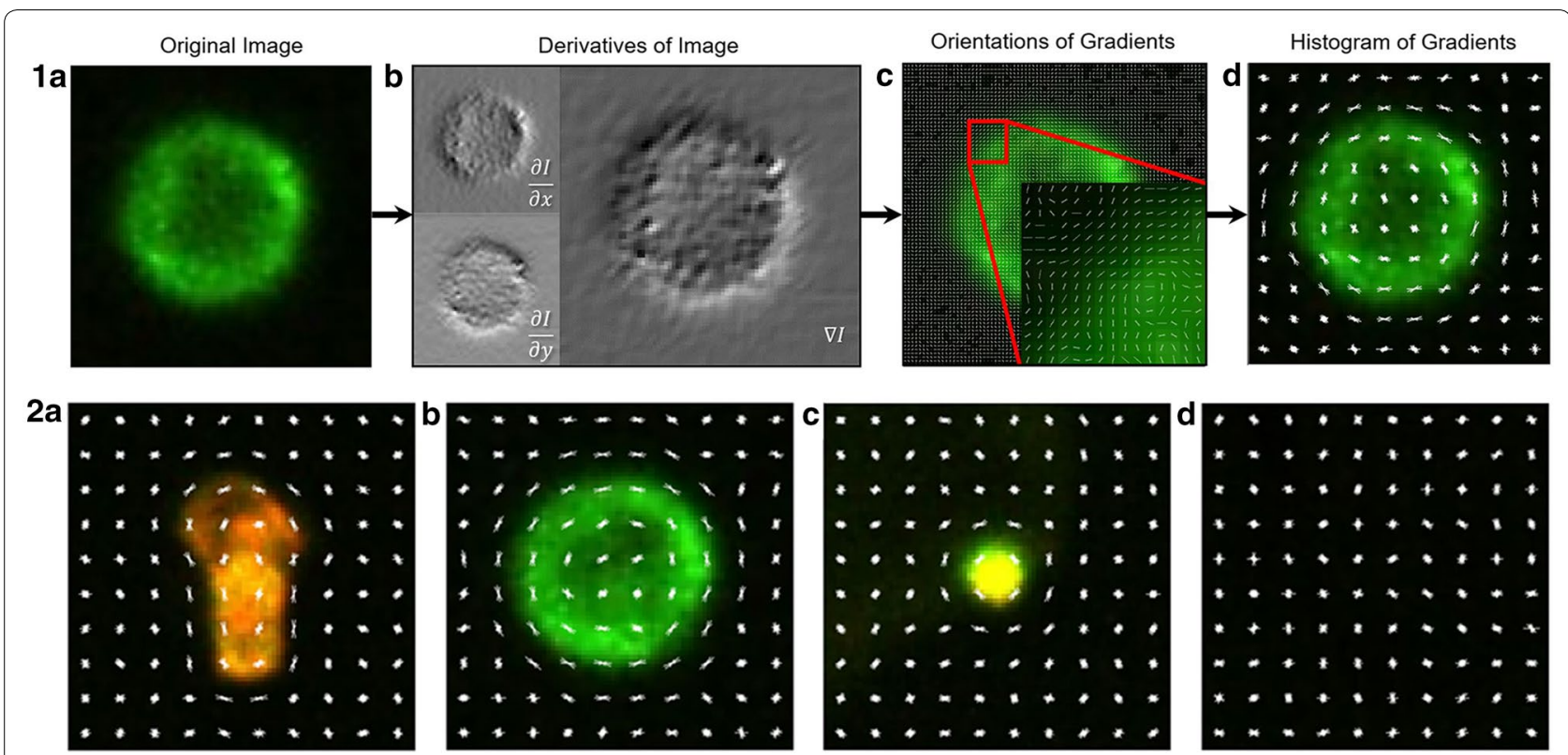

Fig. 4 Original image $\mathbf{1}(\mathrm{a})$, horizontal and vertical derivatives of image $\mathbf{1}(\mathrm{b})$, orientations of gradients overlaid on each pixel $\mathbf{1}(\mathrm{c})$, orientations gathered into histograms and block normalized $\mathbf{1}(\mathrm{d})$. Histogram of gradient features overlaid on cells $\mathbf{2}(\mathrm{a}, \mathrm{b})$, noise $\mathbf{2}(\mathrm{c})$ and background image $\mathbf{2}(\mathrm{d})$

64 GB RAM). We have used OpenCV libraries for HOG to perform GPU processing. Figure 6 shows the results of an example detection, although cells are of different morphology and size, all cells have been detected.

\section{Discussion}

Several methods exist for counting the cells in an image $[24,25]$. The difficulty of performing the cell segmentation or detection task and choosing the correct algorithm depends much on the type of cells being targeted. If the cells are well separated from each other and have uniform intensity, simple thresholding or watershed algorithms are popular choices of approach. If the cells are packed together, algorithms which account for cell shape and size are preferred [26]. But in our application, the unavoidable uneven illumination over time and across the whole microfluidic chip makes the cells fluorescence intensity differs from image to image. And the cells' shape and size were deformed by pillar structure. The intensity, shape and size features could not be utilized directly to detect cells in our pillar-based microfluidic chip system. Another approach, like the one used in this study, is to recognize cells using training-based machine learning methods. Examples of cells and background are shown to the detector, which then learns their most important characteristics. Furthermore, to detect both T-cells and B-cells in a single experiment, the emission spectrum crosstalk of these two cells' dyes should also be overcome. In this work, cell detection from fluorescence microscope images is studied using both HOG and color distribution features with SVM learning classifier. HOG features were utilized to distinguish cells from background noise. But HOG features could only distinguish cells from background and noise, how to eliminate the crosstalk between FITC and PE emission spectrum is still a question. We evaluated the color distribution of cells solely stained by FITC or PE and trained the classifier based on the single stained cells' color features. The classifier was used to determine the cell type when sample were stained by dual-color fluorescence (FITC and PE).

In our work, detection of T-cells and B-cells are important in terms of ratio in between them, hence it is more important to have small ratio of false positives than having small ratio of miss rates. Therefore, we set our detector to have less false positives. This is the reason we chose to prioritize having a high enough specificity such as $99 \%$ while sacrificing accuracy and sensitivity which were $94 \%$ and $90 \%$ respectively. For one single experiment, the total experiment time on microfluidic chip takes about $33 \mathrm{~min}$, which was calculated as follows. Staining the sample requires $15 \mathrm{~min}$, introducing $1 \mu \mathrm{L}$ peripheral blood and $30 \mu \mathrm{L}$ sheath liquid with $10 \mu \mathrm{L} /$ min flow speed takes about $3 \mathrm{~min}$, the whole chip scan costs $15 \mathrm{~min}$, image processing can be simultaneously performed while scanning. FACS requires red blood cell lysis for sample preparation, therefore FACS needs time for the experiment. Compared to the time FACS need ( $>1 \mathrm{~h}$ ), the microfluidic chip requires less time to separate leukocytes directly from whole blood (no lysis). For specific cells types, such as total lymphocytes or T-cells and B-cells, the 


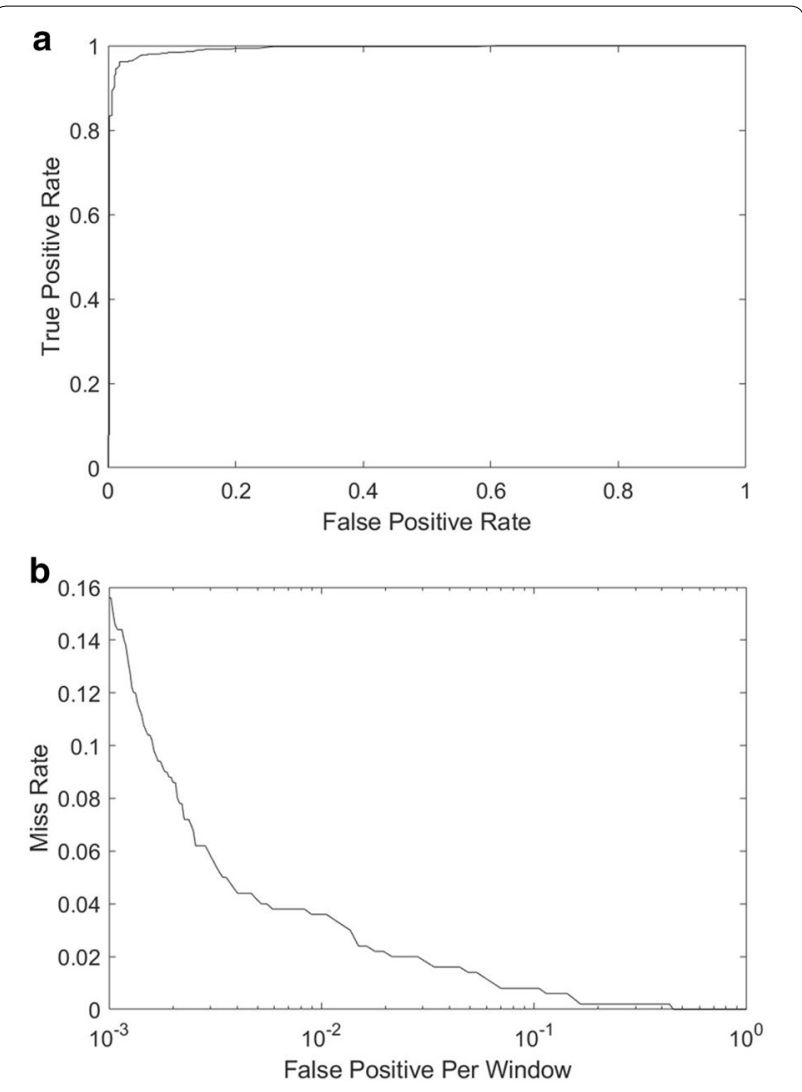

Fig. 5 ROC (a) and DET (b) curves for HOG detector

microfluidic counting system provided a fast and robust way for cell isolation and detection. Although, comparing

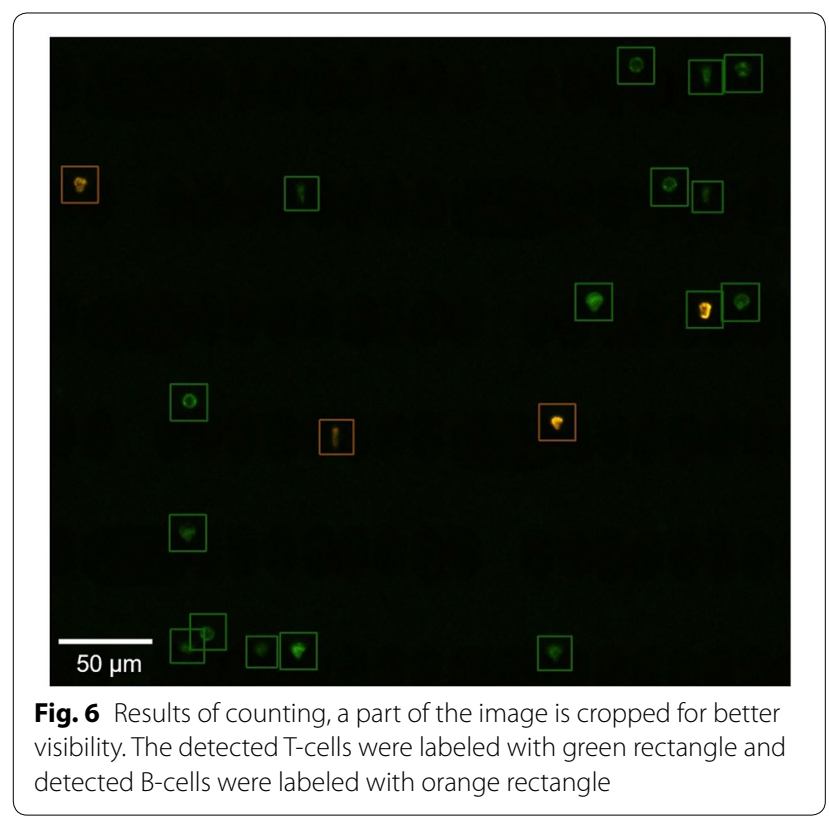

to expensive FACS systems, there are still problems (clogging and spectrum overlap) in multi-type cells sorting for current microfluidic system.

\section{Conclusion}

A pillar-based microfluidic chip with machine learning algorithm for T-cells and B-cells isolation and detection were studied in this paper. A HOG and color features based SVM classifier was proposed to overcome the difficulties in cell detection process, such as fluorescence emission spectrum crosstalk, variability of cell size and morphology, differences in illumination and inconsistence fluorophores expressing level on living cells. We have performed numerous cell detection experiments using the detection technique provided here. The experiment tests performed on various image datasets, have produced satisfactory detection results that prove the effectiveness of our proposed approach. It has achieved a high accuracy of $94 \%$, specificity of $99 \%$ and sensitivity of 90\%. For T-cells B-cells detection we have achieved 96\% cross-validation accuracy. The proposed method and system could also be applied to all other specific leukocytes using different stain agent. Compared to current commercial cell sorter, our system is low cost, small size and microfluidic chip is disposable, it has great potential as a tool for point-of-care cell sorting and analysis.

\section{Authors' contributions}

TM, BT, WL, KH and AMN performed experiments and analysis. TM and BT prepared Figs. TM, BT and FA contributed to write the manuscript text. TM, TIS, YM and FA designed the study. All authors read and approved the final manuscript.

\section{Author details}

${ }^{1}$ Department of Micro-Nano Mechanical Science and Engineering, Graduate School of Engineering, Nagoya University, 1 Furo-cho, Chikusa-ku, Nagoya 464-8603, Japan. ${ }^{2}$ School of Chemistry and Chemical Engineering, Beijing Institute of Technology, Beijing, China. ${ }^{3}$ School of Mechatronic Engineering, University Malaysia Perlis, Perlis, Malaysia. ${ }^{4}$ National Hospital Organization Nagoya Medical Center, Nagoya, Japan.

\section{Acknowledgements}

Not applicable.

\section{Competing interests}

The authors declare that they have no competing interests.

\section{Availability of data and materials}

All relevant data are within the paper.

\section{Funding}

This work was supported in part by the Japan Society for the Promotion of Science (JSPS), Project No.: 15F15757, and supported in part by START Program from Japan Science and Technology Agency, JST.

\section{Publisher's Note}

Springer Nature remains neutral with regard to jurisdictional claims in published maps and institutional affiliations. 
Received: 31 July 2018 Accepted: 20 September 2018

Published online: 16 October 2018

\section{References}

1. Costa S, Schutz S, Cornec D et al (2016) B-cell and T-cell quantification in minor salivary glands in primary Sjögren's syndrome: development and validation of a pixel-based digital procedure. Arthritis Res Ther 18(1):21

2. Huang X, Guo J, Wang X et al (2014) A contact-imaging based microfluidic cytometer with machine-learning for single-frame super-resolution processing. PLoS ONE 9(8):e104539

3. Hassan U Jr, Reddy B Jr, Damhorst G et al (2015) A microfluidic biochip for complete blood cell counts at the point-of-care. Technology 3(4):201

4. Piyasena ME, Graves SW (2014) The intersection of flow cytometry with microfluidics and microfabrication. Lab Chip 14(6):1044-1059

5. El-Ali J, Sorger PK, Jensen KF (2006) Cells on chips. Nature 442(7101):403-411

6. Shields CW IV, Reyes CD, López GP (2015) Microfluidic cell sorting: a review of the advances in the separation of cells from debulking to rare cell isolation. Lab Chip 15(5):1230-1249

7. Wu HW, Hsu RC, Lin CC et al (2010) An integrated microfluidic system for isolation, counting, and sorting of hematopoietic stem cells. Biomicrofluidics 4(2):989

8. Agarwal S, Sebastian A, Forrester LM et al (2012) Formation of embryoid bodies using dielectrophoresis. Biomicrofluidics 6(2):24101

9. Hoi SK, Kim HV, Huy MN, Sow C-H, Ow YS, Bettiol AA (2010) Passive optical separation and enrichment of cells by size difference. Biomicrofluidics 4:044111

10. Kim Y, Lee SH, Kim B (2009) Droplet-based magnetically activated cell separation: analysis of separation efficiency based on the variation of flow-induced circulation in a pendent drop. Anal Bioanal Chem 395(7):2415-2421

11. Hassan U, Reddy B Jr, Damhorst G et al (2015) A microfluidic biochip for complete blood cell counts at the point-of-care. Technology 3(04):201-213

12. Nam J, Lim H, Kim C et al (2012) Density-dependent separation of encapsulated cells in a microfluidic channel by using a standing surface acoustic wave. Biomicrofluidics 6(2):24120(1-10)

13. Liu Y, Hartono D, Lim KM (2012) Cell separation and transportation between two miscible fluid streams using ultrasound. Biomicrofluidics 6(1):12802(1-14)
14. Masuda T, Song W, Nakanishi H et al (2017) Rare cell isolation and recovery on open-channel microfluidic chip. PLoS ONE 12(4):e0174937

15. Hosokawa M, Asami M, Nakamura S et al (2012) Leukocyte counting from a small amount of whole blood using a size-controlled microcavity array. Biotechnol Bioeng 109(8):2017

16. Alvankarian J, Bahadorimehr A, Yeop MB (2013) A pillar-based microfilter for isolation of white blood cells on elastomeric substrate. Biomicrofluidics 7(1):142-502

17. Ji HM, Samper $V$, Chen $Y$ et al (2008) Silicon-based microfilters for whole blood cell separation. Biomed Microdevice 10(2):251-257

18. Buggenthin F, Marr C, Schwarzfischer M et al (2013) An automatic method for robust and fast cell detection in bright field images from high-throughput microscopy. BMC Bioinform 14(1):1-12

19. Faustino GM, Gattass M, Rehen S et al (2009) Automatic embryonic stem cells detection and counting method in fluorescence microscopy images. In: Proceedings of IEEE international conference on symposium on biomedical imaging, pp 799-802

20. Dalal N, Triggs B (2005) Histograms of oriented gradients for human detection. In: Proceedings of computer vision and pattern recognition, pp 886-893

21. Suykens JA, Vandewalle J (1999) Least squares support vector machine classifiers. Neural Process Lett 9(3):293-300

22. Noor AM, Masuda T, Lei W, Horio K, Miyata Y, Namatame M, Hayase Y, Saito Tl, Arai F (2018) A microfluidic chip for capturing, imaging and counting CD3+ T-lymphocytes and CD19+ B-lymphocytes from whole blood. Sens Actuators B Chem 276:107-113

23. Otsu N (1979) A threshold selection method from gray-level histograms. IEEE Trans Syst Man Cybern 9(1):62-66

24. Kamentsky L, Jones TR, Fraser A, Bray MA, Logan DJ, Madden KL, Ljosa V, Rueden C, Eliceiri KW, Carpenter AE (2011) Improved structure, function and compatibility for CellProfiler: modular high-throughput image analysis software. Bioinformatics 27(8):1179-1180

25. Wiesmann V, Franz D, Held C, Münzenmayer C, Palmisano R, Wittenberg $T$ (2015) Review of free software tools for image analysis of fluorescence cell micrographs. J Microsc 257(1):39-53

26. Xie W, Noble JA, Zisserman A (2018) Microscopy cell counting and detection with fully convolutional regression networks. Comput Methods Biomech Biomed Eng Imaging Vis 6(3):283-292

\section{Submit your manuscript to a SpringerOpen ${ }^{\circ}$ journal and benefit from:}

- Convenient online submission

- Rigorous peer review

- Open access: articles freely available online

- High visibility within the field

Retaining the copyright to your article

Submit your next manuscript at springeropen.com 